\title{
Age and Retinal Nerve Fiber Layer Thickness Measured by Spectral Domain Optical Coherence Tomography
}

\author{
Jong Yeon Lee, Young Hoon Hwang ${ }^{2,3}$, Sun Min Lee ${ }^{4}$, Yong Yeon Kim ${ }^{5}$ \\ ${ }^{1}$ Department of Ophthalmology, Gachon University Gil Hospital, Incheon, Korea \\ ${ }^{2}$ Department of Ophthalmology, Kim's Eye Hospital, Myung-Gok Eye Research Institute, \\ Konyang University College of Medicine, Seoul, Korea \\ ${ }^{3}$ Department of Ophthalmology, Armed Forces Capital Hospital, Seongnam, Korea \\ ${ }^{4}$ Department of Radiation Oncology, Korea University College of Medicine, Seoul, Korea \\ ${ }^{5}$ Department of Ophthalmology, Korea University College of Medicine, Seoul, Korea
}

\begin{abstract}
Purpose: To evaluate the association between age and peripapillary retinal nerve fiber layer (RNFL) thickness measured by Cirrus high-definition (HD) spectral domain optical coherence tomography (OCT) in healthy Korean subjects.

Methods: A total of 302 eyes from 155 healthy Korean subjects (age range, 20 to 79 years) underwent RNFL thickness measurements using the Cirrus HD-OCT. Average, quadrant, and clock-hour RNFL thickness parameters were analyzed in terms of age using linear mixed effect models.

Results: Average RNFL demonstrated a slope of $-2.1 \mu \mathrm{m}$ per decade of age $(p<0.001)$. In quadrant analysis, superior $(-3.4 \mu \mathrm{m} /$ decade, $p<0.001)$ and inferior $(-2.9 \mu \mathrm{m} /$ decade, $p<0.001)$ quadrants showed steeper slopes, whereas temporal $(-1.1 \mu \mathrm{m} /$ decade, $p<0.001)$ and nasal $(-1.0 \mu \mathrm{m} / \mathrm{decade}, p<0.001)$ quadrants revealed shallower slopes. Among the 12 clock-hour sectors, clock hours $6(-4.5 \mu \mathrm{m} / \mathrm{decade}, p<0.001)$ and $1(-4.1$ $\mu \mathrm{m} /$ decade, $p<0.001)$ showed the greatest tendency to decline with age; RNFLs of the $3(-0.2 \mu \mathrm{m} / \mathrm{decade}, p$ $=0.391)$ and $4(-0.6 \mu \mathrm{m} /$ decade, $p=0.052)$ o'clock hour sectors did not show significant decay.

Conclusions: RNFL thickness was associated with age, especially in superior and inferior areas. The topographic distribution of correlation between age and RNFL thickness was not uniform.
\end{abstract}

Key Words: Aging, Glaucoma, Optical coherence tomography

As glaucoma is associated with progressive retinal nerve fiber layer (RNFL) thinning, measuring RNFL thickness over time is essential for the evaluation of glaucoma [1-3]. It has been reported that RNFL thinning is also associated with aging [4-21]. Considering both age and glaucoma cause progressive RNFL thinning, it is important to assess the rate and topographic distribution of RNFL thinning associated with age for the accurate evaluation of glaucoma.

The association between age and RNFL thickness in

Received: December 6, 2010 Accepted: April 18, 2011

Corresponding Author: Young Hoon Hwang, MD. Department of Ophthalmology, Kim's Eye Hospital, \#136 Yeongsin-ro, Yeongdeungpo-gu, Seoul 150-034, Korea. Tel: 82-2-2639-7777, Fax: 82-2-2633-3976, E-mail: brainh@hanmail.net healthy subjects has been investigated by histological methods [4-8], scanning laser polarimetry [9-12], and optical coherence tomography (OCT) [13-22]. Various studies with time-domain OCT have reported that the slope of average RNFL thickness ranged from -1.6 to $-3.3 \mu \mathrm{m}$ per decade of age, with inconsistent results for topographic distribution of RNFL slopes [13-22]. RNFL thickness measured by time-domain OCT was reported to be different from that measured by spectral domain OCT $[23,24]$. In addition, there were differences in RNFL thickness according to ethnicity [15]. However, to date, little is known about the association between age and RNFL thickness measured by spectral-domain OCT in Korean subjects.

This study was performed to evaluate the association between age and RNFL thickness measured by the Cirrus 
high-definition (HD) spectral-domain OCT (Cirrus HDOCT; Carl Zeiss Meditec, Dublin, CA, USA) in healthy Korean subjects.

\section{Materials and Methods}

The study protocol was approved by the institutional review board of the Armed Forces Capital Hospital, Korea. All procedures conformed to the Declaration of Helsinki, and all participants provided informed consent before enrollment. Among those who underwent annual ocular examinations at the Armed Forces Capital Hospital, Korea, healthy volunteers (including ranks, officers, and reserve officers) were recruited from June 2009 to August 2010.

Each participant underwent a full ophthalmic examination, including the assessment of visual acuity, refractive error by autorefractokeratometer (RK-F1; Canon, Tokyo, Japan), intraocular pressure (IOP) with a non-contact tonometer (Topcon CT-80; Topcon, Tokyo, Japan), automated visual field test with the Humphrey visual field analyzer (Carl Zeiss Meditec), optic nerve head evaluation and fundus examination with a 90 diopter lens, and peripapillary RNFL thickness measurement with a Cirrus HD-OCT.

Inclusion criteria were as follows: best-corrected visual acuity of $20 / 25$ or better, spherical equivalent (spherical refractive error $+1 / 2$ cylindrical refractive error in the negative form) within \pm 2.0 diopters, normal IOP $(<21 \mathrm{mmHg})$, normal visual field (glaucoma hemifield test within normal limits and pattern standard deviation probability greater than 5\%) with reliable results (false-positive or false-nega- tive rate $\leq 15 \%$ or fixation losses $\leq 20 \%$ ), normal optic nerve head without glaucomatous changes (i.e., increased cup-todisc ratio, narrowing of neuroretinal rim), and no retinal pathology. Subjects with a history of systemic diseases including hypertension and diabetes, previous ocular trauma or surgery, or peripapillary atrophy extending more than 1.7 $\mathrm{mm}$ from the center of the optic disc were excluded.

A $200 \times 200$ cube optic disc scan was obtained with the Cirrus HD-OCT without pupil dilation. Using the iris and fundus viewports, the alignment was properly positioned to the optic nerve head in the center of the scan. Once the optic nerve head was centered on the live scanning laser image, a $6 \times 6$-mm square of data was captured. Only images without a prominent involuntary saccade during the scan and signal strength $\geq 8$ were included. Using the glaucoma OU analysis mode of the Cirrus HD-OCT (software ver. 4.5.1.11), the average, superior, nasal, inferior, temporal, and 12 clock-hour RNFL thickness parameters were obtained.

Linear mixed effects models were fitted to the RNFL thickness to evaluate its association with age. The absolute slopes of RNFL changes in average, superior, nasal, inferior, temporal, and 12 clock-hour sectors were evaluated. Because the rate of change can be affected by the level of measurement, the slope was normalized by calculating the slope divided by the average parameter value to evaluate whether the relative rate of change was homogenous throughout the various sectors [18]. The relationship among the absolute and relative (normalized) slopes and average RNFL thickness of each clock-hour sector was evaluated

Table 1. Mean \pm standard deviation of retinal nerve fiber layer thickness $(\mu \mathrm{m})$ on average, quadrants, and clock-hour sectors in different age groups

\begin{tabular}{lccccc}
\hline & $20-29 \mathrm{yr}$ & $30-39 \mathrm{yr}$ & $40-49 \mathrm{yr}$ & $50-59 \mathrm{yr}$ & $60-79 \mathrm{yr}$ \\
\hline No. of eyes & 69 & 62 & 62 & 56 & 53 \\
Average & $99.04 \pm 4.20$ & $98.19 \pm 5.29$ & $94.44 \pm 4.09$ & $92.63 \pm 4.80$ & $89.60 \pm 4.73$ \\
Superior & $129.81 \pm 10.12$ & $125.66 \pm 10.37$ & $119.73 \pm 10.11$ & $116.93 \pm 9.21$ & $114.42 \pm 9.48$ \\
Nasal & $71.45 \pm 6.72$ & $71.27 \pm 6.76$ & $68.71 \pm 5.48$ & $68.00 \pm 6.78$ & $67.02 \pm 5.61$ \\
Inferior & $127.38 \pm 8.76$ & $126.53 \pm 10.95$ & $122.89 \pm 9.22$ & $118.80 \pm 10.72$ & $114.38 \pm 9.75$ \\
Temporal & $67.62 \pm 6.24$ & $69.44 \pm 7.19$ & $66.43 \pm 5.97$ & $66.39 \pm 5.11$ & $62.23 \pm 8.07$ \\
Clock-hour sector & & & & $123.81 \pm 18.10$ & $122.34 \pm 20.74$ \\
12 & $138.96 \pm 17.04$ & $127.90 \pm 19.19$ & $125.11 \pm 21.48$ & 10 & $103.06 \pm 14.98$ \\
1 & $120.93 \pm 18.74$ & $115.76 \pm 17.20$ & $109.61 \pm 16.32$ & $104.79 \pm 15.24$ & $80.70 \pm 11.00$ \\
2 & $90.10 \pm 13.02$ & $88.58 \pm 13.45$ & $83.19 \pm 11.80$ & $81.64 \pm 11.10$ & $58.57 \pm 8.20$ \\
3 & $59.57 \pm 8.40$ & $60.92 \pm 8.00$ & $59.54 \pm 8.20$ & $59.27 \pm 6.67$ & $61.98 \pm 6.49$ \\
4 & $64.71 \pm 9.04$ & $64.37 \pm 8.09$ & $63.55 \pm 8.00$ & $62.77 \pm 7.90$ & $90.68 \pm 12.79$ \\
5 & $102.33 \pm 15.58$ & $97.48 \pm 13.19$ & $96.66 \pm 13.11$ & $95.02 \pm 11.90$ & $125.60 \pm 20.40$ \\
6 & $144.59 \pm 17.22$ & $140.16 \pm 19.40$ & $135.42 \pm 19.17$ & $127.50 \pm 20.67$ & $126.87 \pm 17.89$ \\
7 & $136.60 \pm 12.52$ & $141.85 \pm 17.13$ & $135.26 \pm 15.19$ & $134.00 \pm 14.87$ & $64.28 \pm 10.48$ \\
8 & $69.54 \pm 10.00$ & $70.94 \pm 10.72$ & $68.75 \pm 7.28$ & $68.63 \pm 8.49$ & $51.49 \pm 6.96$ \\
9 & $55.00 \pm 6.10$ & $56.55 \pm 5.89$ & $53.66 \pm 5.19$ & $53.59 \pm 5.59$ & $71.06 \pm 10.19$ \\
10 & $78.29 \pm 8.01$ & $80.81 \pm 9.32$ & $76.94 \pm 7.65$ & $76.84 \pm 10.64$ & $116.34 \pm 15.73$ \\
\hline
\end{tabular}


Table 2. Absolute and normalized slopes of retinal nerve fiber layer thickness on average, quadrants, and clock-hour sectors

\begin{tabular}{lcccc}
\hline & Absolute slope $(\mu \mathrm{m} /$ decade of year $)$ & $95 \%$ confidence interval & $p_{\text {-value }}^{*}$ & Normalized slope $^{*}$ \\
\hline Average & -2.05 & -2.39 to -1.72 & $<0.001$ & -0.02156 \\
Superior & -3.40 & -4.11 to -2.70 & $<0.001$ & -0.02792 \\
Nasal & -0.97 & -1.41 to -0.53 & $<0.001$ & -0.01397 \\
Inferior & -2.88 & -3.57 to -2.18 & $<0.001$ & -0.02353 \\
Temporal & -1.08 & -1.55 to -0.61 & $<0.001$ & -0.01622 \\
Clock-hour sectors & & & & \\
12 & -3.16 & -4.54 to -1.79 & $<0.001$ & -0.02467 \\
1 & -4.05 & -5.22 to -2.87 & $<0.001$ & -0.03635 \\
2 & -2.09 & -2.95 to -1.23 & $<0.001$ & -0.02454 \\
3 & -0.23 & -0.77 to 0.30 & 0.391 & -0.00386 \\
4 & -0.56 & -1.12 to 0.01 & 0.052 & -0.00881 \\
5 & -2.28 & -3.23 to -1.34 & $<0.001$ & -0.02356 \\
6 & -4.52 & -5.87 to -3.18 & $<0.001$ & -0.03341 \\
7 & -1.82 & -2.93 to -0.70 & 0.002 & -0.01346 \\
8 & -0.89 & -1.56 to -0.22 & 0.010 & -0.01298 \\
9 & -0.81 & -1.23 to -0.39 & $<0.001$ & -0.01495 \\
10 & -1.52 & -2.17 to -0.87 & $<0.001$ & -0.01974 \\
\hline
\end{tabular}

${ }^{*} p$-value for the absolute slope difference from a zero slope.

by Spearman's rho test. Significance was set at $p<0.05$. All statistical analysis of the data was performed using SPSS ver. 12.0 (SPSS Inc., Chicago, IL, USA).

\section{Results}

A total of 302 eyes of 155 healthy Korean male participants were enrolled. The mean \pm standard deviation age and spherical equivalent were $42.90 \pm 16.15$ years (range, 20 to 79 years) and $-0.52 \pm 0.88$ diopters (range, -1.88 to 1.75 diopters), respectively.

Overall subjects were divided into 5 groups according to the age. RNFL thicknesses of average, quadrants, and clock-hour sectors in each age group are listed in Table 1. The results of the RNFL thickness slope analysis are listed in Table 2. The average RNFL demonstrated a slope of -2.1 $\mu \mathrm{m}$ per decade of age $(p<0.001$; normalized, -0.022$)$. In quadrant analysis, the superior $(-3.4 \mu \mathrm{m} /$ decade; $p<0.001$; normalized, -0.028) and inferior $(-2.9 \mu \mathrm{m} /$ decade; $p<0.001$; normalized, -0.024$)$ quadrants showed greatest tendency to decrease with age, whereas the temporal $(-1.1 \mu \mathrm{m} / \mathrm{decade}$; $p<0.001$; normalized, -0.016) and nasal (-1.0 $\mu \mathrm{m} /$ decade; $p$ $<0.001$; normalized, -0.014) quadrants revealed shallower slopes of decay. The slopes of superior and inferior quadrants were significantly greater than the slopes of nasal and temporal quadrants $(p<0.001)$; comparisons of slopes between nasal and temporal, superior and inferior quadrants were not significant $(p>0.20)$.

Among the 12 clock-hour sectors, clock hours 6 (-4.5 $\mu \mathrm{m} /$ decade; $p<0.001$; normalized, -0.033$)$ and $1(-4.1 \mu \mathrm{m} /$ decade; $p<0.001$; normalized, -0.036) showed the greatest tendency to decline with age; RNFLs of the $3(-0.2 \mu \mathrm{m} / \mathrm{de}-$ cade; $p=0.391$; normalized, -0.004$)$ and 4 (-0.6 $\mu \mathrm{m} /$ decade; $p=0.052$; normalized, -0.009) o'clock hour sectors did not show significant decay. Other sectors $(12,2,5,7,8,9,10$, and 11 o'clock hour sectors) also showed significant decay with age $(p \leq 0.010)$.

The relationship between the RNFL thickness slope and average RNFL thickness of each clock-hour sector is presented in Fig. 1. Both absolute and normalized slopes in RNFL thickness were significantly associated with average RNFL thickness of each sector (Spearman's rho $=0.853, p$ $<0.001$ for absolute slope; Spearman's rho $=0.661, p=0.018$ for normalized slope).

\section{Discussion}

In the present study, peripapillary RNFL thickness measured by the Cirrus HD-OCT was associated with age. The correlations were prominent in superior and inferior areas, whereas those in nasal and temporal areas were less remarkable.

Various studies have investigated the correlation between age and RNFL thickness. Cross-sectional histologic studies revealed that the optic nerve of a normal subject loses its axons in the range of 4,900 to 6,700 per year [4-7]. However, histologic methods have limitations of artifacts caused by tissue swelling, autolysis, shrinkage, or differences in sampling location [8].

Regarding in vivo methods, studies with scanning laser 

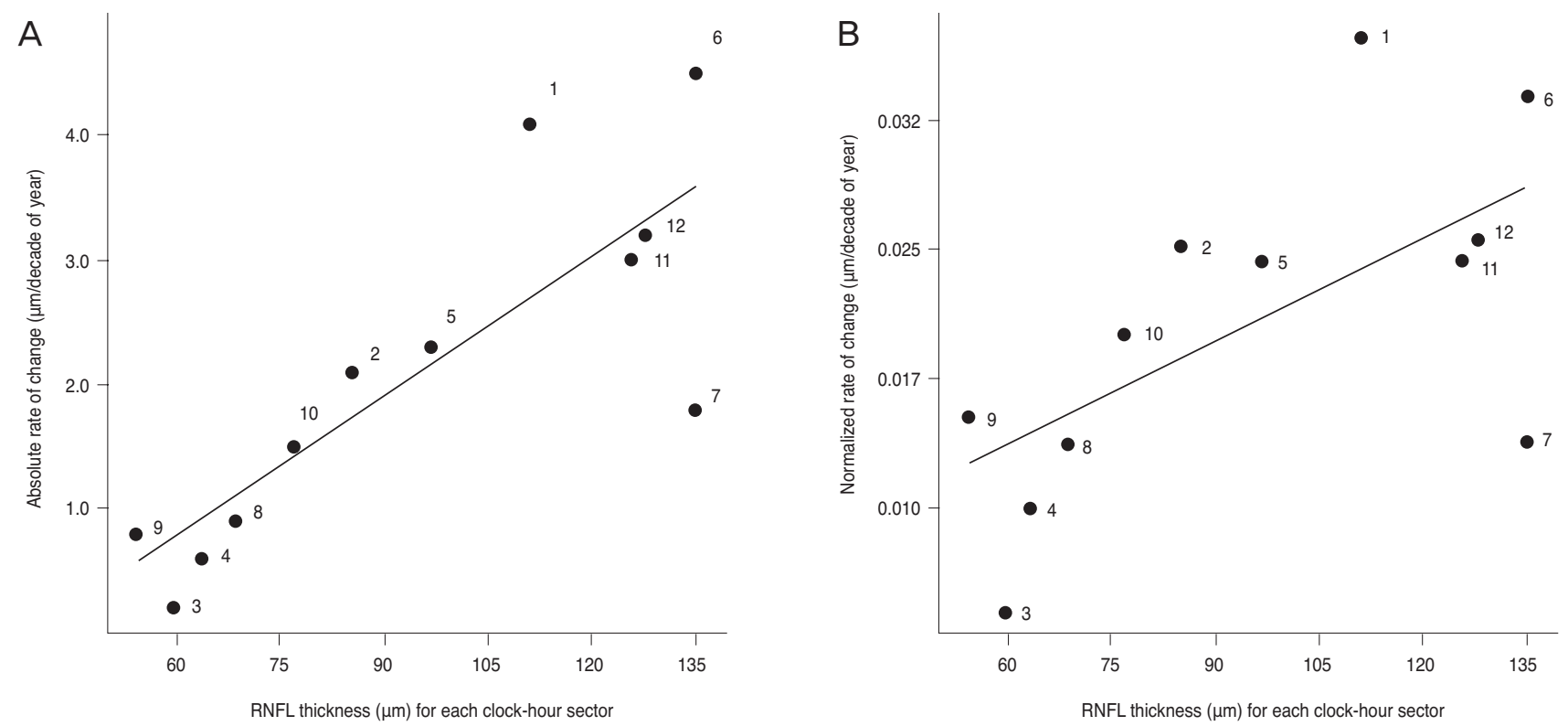

Fig. 1. The relationship between absolute (A), normalized (B) slopes of retinal nerve fiber layer (RNFL) thickness, and RNFL thickness of each clock-hour sector (numbers indicate the clock-hour sectors). Both absolute and normalized rates of RNFL thickness slopes were significantly associated with the average RNFL thickness of each sector (Spearman's rho $=0.853, p<0.001$ for absolute slope; Spearman's rho $=0.661, p=0.018$ for normalized slope).

polarimetry and OCT have been reported [9-22]. In crosssectional studies with scanning laser polarimetry, the average RNFL thickness decay ranged from -0.8 to $-5.0 \mu \mathrm{m}$ per decade of age [9-12]. However, topographic patterns of decrease in RNFL were different between the studies: one study showed only significant changes in nasal and inferior areas [9], whereas another study revealed a faster rate of change in the superior area compared to the inferior area [12].

Various cross-sectional studies with OCT reported the relationship between age and RNFL thickness [13-22]. The reported rates of RNFL thickness change ranged from -1.6 to $-3.3 \mu \mathrm{m}$ per decade of age, which includes our outcome $(-2.1 \mu \mathrm{m})$. However, in terms of topographic patterns of RNFL slope, inconsistent results were found among the studies using OCT [13-21]. To date, the results of 3 studies with Stratus OCT (Carl Zeiss Meditec) are available for comparison of the average, quadrant, and clock-hour sector slopes of RNFL changes with 95\% confidence intervals (Table 3). A study by Parikh et al. [16] showed that the average RNFL thickness slope was $-1.6 \mu \mathrm{m}$ per decade of age; the rates of the superior and inferior quadrants were -2.3 and $-0.9 \mu \mathrm{m}$ per decade of age, respectively. This finding was in agreement with the study results of Feuer et al. [17] who reported that the average slope was $-2.4 \mu \mathrm{m}$ per decade of age, and those of superior and inferior quadrants were -4.3 and $-1.5 \mu \mathrm{m}$ per decade of age, respectively. Both of these studies showed a shallower slope of RNFL changes in the inferior area. Parikh et al. [16] suggested the axons of the inferior RNFL are more resistant to agerelated changes than axons of the superior area.

On the contrary, in another study using Stratus OCT, Sung et al. [18] reported that average RNFL slope was -2.6 $\mu \mathrm{m}$ per decade of age, and both superior and inferior quadrant RNFLs showed the greatest slopes $(-3.5$ and $-3.6 \mu \mathrm{m}$ per decade of age, respectively) which was in line with the present study results. The slope of the inferior area in our study results was steeper than those of other studies $[16,17]$ as presented in Table 3. For instance, the $95 \%$ confidence interval of the clock-hour 6 slope in the present study did not overlap with those of previous studies [16,17], which may imply significant differences. Other studies had differing findings, demonstrating similar RNFL slopes in all regions [19], significant slopes at superior, inferior, and temporal areas [20], or the steepest slope in the inferiortemporal and superior-temporal areas $[21,22]$. These discrepancies may be explained a part by differences in ethnicity, refractive error, or imaging device between the present study and other studies.

In the present study, the topographic distribution of RNFL change with age was not uniform: the slopes of superior and inferior areas (except for the 7 o'clock hour sector) were significantly steeper than those of the nasal and temporal areas. This topographic difference in RNFL changes may be explained by two hypotheses. First, an area with greater axonal diameter may have a steeper decline than an area with thinner axons. In the present study, areas with greater RNFL thickness had steeper slopes, as 
Table 3. Slopes of retinal nerve fiber layer thickness in the present study and previous studies ( $\mu \mathrm{m} / \mathrm{decade}$ of age, $95 \%$ confidence interval)

\begin{tabular}{lllll}
\hline & Present study & Sung et al. [18] & Parikh et al. [16] & Feuer et al. [17] \\
\hline Average & $-2.1(-2.4,-1.7)$ & $-2.6(-4.4,-0.7)$ & $-1.6(-2.9,-0.2)$ & $-2.4(-3.1,-1.8)$ \\
Superior & $-3.4(-4.1,-2.7)$ & $-3.5(-5.3,-1.6)$ & $-2.3(-3.7,-0.9)$ & $-4.3(-5.3,-3.3)$ \\
Nasal & $-1.0(-1.4,-0.5)$ & $-2.5(-4.3,-0.6)$ & $-1.1(-2.5,0.4)$ & $-2.9(-3.9,-1.8)$ \\
Inferior & $-2.9(-3.6,-2.2)$ & $-3.6(-5.4,-1.8)$ & $-0.9(-2.3,0.6)$ & $-1.5(-2.5,-0.4)$ \\
Temporal & $-1.1(-1.5,-0.6)$ & $-0.7(-2.5,1.2)$ & $-2.0(-3.4,-0.7)$ & $-1.0(-1.8,-0.2)$ \\
Clock-hour sector & & & \\
12 & $-3.2(-4.5,-1.8)$ & $-3.6(-5.5,-1.8)$ & $-1.9(-3.3,-0.5)$ & $-4.5(-6.0,-3.0)$ \\
1 & $-4.1(-5.2,-2.9)$ & $-4.5(-6.3,-2.6)$ & $-3.2(-4.6,-1.8)$ & $-5.4(-6.8,-4.1)$ \\
2 & $-2.1(-3.0,-1.2)$ & $-3.6(-5.5,-1.8)$ & $-1.1(-2.5,0.4)$ & $-3.9(-5.2,-2.5)$ \\
3 & $-0.2(-0.8,0.3)$ & $-1.8(-3.7,0)$ & $-0.5(-1.9,1.0)$ & $-2.0(-3.0,-0.9)$ \\
4 & $-0.6(-1.1,0)$ & $-1.9(-3.7,0)$ & $-0.9(-2.3,0.5)$ & $-2.7(-3.9,-1.5)$ \\
5 & $-2.3(-3.2,-1.3)$ & $-3.9(-5.7,-2.0)$ & $-0.4(-1.9,1.0)$ & $-1.1(-2.6,0.4)$ \\
6 & $-4.5(-5.9,-3.2)$ & $-3.9(-5.7,-2.0)$ & $-0.2(-1.7,1.2)$ & $-0.9(-2.7,0.7)$ \\
7 & $-1.8(-2.9,-0.7)$ & $-3.1(-4.9,-1.3)$ & $-2.3(-3.7,-0.8)$ & $-2.4(-3.8,-1.0)$ \\
8 & $-0.9(-1.6,-0.2)$ & $-1.1(-2.9,0.8)$ & $-2.8(-4.1,-1.4)$ & $-1.4(-2.3,-0.4)$ \\
9 & $-0.8(-1.2,-0.4)$ & $-0.4(-2.2,-1.5)$ & $-1.5(-2.9,-0.1)$ & $-0.6(-1.2,0)$ \\
10 & $-1.5(-2.1,-0.9)$ & $-0.1(-2.5,1.2)$ & $-1.8(-3.2,-0.4)$ & $-1.1(-2.1,0)$ \\
11 & $-3.0(-4.2,-1.8)$ & $-2.3(-4.2,-0.5)$ & $-1.6(-3.0,-0.2)$ & $-3.0(-4.3,-1.6)$ \\
\hline
\end{tabular}

shown in Fig. 1. A histologic study by Mikelberg et al. [25] documented that the superior-nasal area had axons with greatest diameter and the temporal area had thinner axons. Therefore, the steepest slope of the 1 o'clock hour sector (superior-nasal area) and shallower slope of the temporal area in the present study may be explained by this hypothesis. Second, if axonal loss does not occur with a homogeneous topographic distribution, areas resistant to axonal loss with age (i.e., inferior area $[16,17]$ or temporal area [18] in the previous studies) may have shallower slopes than other areas. In the present study, the 7 o'clock hour sector had a shallower slope than other clock-hour sectors with similar RNFL thickness (clock-hour sectors of 6,11, and 12). This may be explained by topographic differences in resistance to age-related changes.

One limitation in this study is that our results were based on cross-sectional data rather than longitudinal data with serial follow-up. Although longitudinal study would provide a more accurate estimate of age-related RNFL change, the long term test-retest repeatability of OCT is warranted. In addition, data from a cross-sectional study can investigate RNFL change over a wide age range. Alternatively, our results are not free from selection bias. Data of this military hospital-based study may not represent population-based results.

In conclusion, RNFL thickness of healthy Korean subjects measured by the Cirrus HD-OCT was associated with age, especially in the superior and inferior areas. The topographic distributions of RNFL slopes were not uniform. These results should be considered for the assess- ment of RNFL thickness over time.

\section{Conflict of Interest}

No potential conflict of interest relevant to this article was reported.

\section{References}

1. Schuman JS, Hee MR, Puliafito CA, et al. Quantification of nerve fiber layer thickness in normal and glaucomatous eyes using optical coherence tomography. Arch Ophthalmol 1995;113:586-96.

2. Lee EJ, Kim TW, Weinreb RN, et al. Trend-based analysis of retin al nerve fiber layer thickness measured by optical coherence tomography in eyes with localized nerve fiber layer defects. Invest Ophthalmol Vis Sci 2011;52:1138-44.

3. Leung CK, Liu S, Weinreb RN, et al. Evaluation of retinal nerve fiber layer progression in glaucoma a prospective analysis with neuroretinal rim and visual field progression. Ophthalmology 2011;118:1551-7.

4. Dolman CL, McCormick AQ, Drance SM. Aging of the optic nerve. Arch Ophthalmol 1980;98:2053-8.

5. Balazsi AG, Rootman J, Drance SM, et al. The effect of age on the nerve fiber population of the human optic nerve. Am J Ophthalmol 1984;97:760-6.

6. Johnson BM, Miao M, Sadun AA. Age-related decline of human optic nerve axon populations. Age 1987;10:5-9.

7. Jonas JB, Muller-Bergh JA, Schlotzer-Schrehardt UM, Naumann GO. Histomorphometry of the human optic nerve. Invest Ophthalmol Vis Sci 1990;31:736-44.

8. Frenkel S, Morgan JE, Blumenthal EZ. Histological measurement of retinal nerve fibre layer thickness. Eye (Lond) 2005;19:491-8. 
9. Chi QM, Tomita G, Inazumi K, et al. Evaluation of the effect of aging on the retinal nerve fiber layer thickness using scanning laser polarimetry. J Glaucoma 1995;4:406-13.

10. Toprak AB, Yilmaz OF. Relation of optic disc topography and age to thickness of retinal nerve fibre layer as measured using scanning laser polarimetry, in normal subjects. Br J Ophthalmol 2000;84:473-8.

11. Poinoosawmy D, Fontana L, Wu JX, et al. Variation of nerve fibre layer thickness measurements with age and ethnicity by scanning laser polarimetry. $\mathrm{Br} J$ Ophthalmol 1997;81:350-4.

12. Da Pozzo S, Iacono P, Marchesan R, et al. The effect of ageing on retinal nerve fibre layer thickness: an evaluation by scanning laser polarimetry with variable corneal compensation. Acta Ophthalmol Scand 2006;84:375-9.

13. Schuman JS, Hee MR, Puliafito CA, et al. Quantification of nerve fiber layer thickness in normal and glaucomatous eyes using optical coherence tomography. Arch Ophthalmol 1995;113:586-96.

14. Varma R, Bazzaz S, Lai M. Optical tomography-measured retinal nerve fiber layer thickness in normal latinos. Invest Ophthalmol Vis Sci 2003;44:3369-73.

15. Budenz DL, Anderson DR, Varma R, et al. Determinants of normal retinal nerve fiber layer thickness measured by Stratus OCT. Ophthalmology 2007;114:1046-52.

16. Parikh RS, Parikh SR, Sekhar GC, et al. Normal age-related decay of retinal nerve fiber layer thickness. Ophthalmology 2007;114:921-6.

17. Feuer WJ, Budenz DL, Anderson DR, et al. Topographic differences in the age-related changes in the retinal nerve fiber layer of normal eyes measured by Stratus optical co- herence tomography. J Glaucoma 2011;20:133-8.

18. Sung KR, Wollstein G, Bilonick RA, et al. Effects of age on optical coherence tomography measurements of healthy retinal nerve fiber layer, macula, and optic nerve head. Ophthalmology 2009;116:1119-24.

19. Mok KH, Lee VW, So KF. Retinal nerve fiber layer measurement of the Hong Kong Chinese population by optical coherence tomography. J Glaucoma 2002;11:481-3.

20. Kanamori A, Escano MF, Eno A, et al. Evaluation of the effect of aging on retinal nerve fiber layer thickness measured by optical coherence tomography. Ophthalmologica 2003;217:273-8.

21. Kanno M, Nagasawa M, Suzuki M, Yamashita H. Peripapillary retinal nerve fiber layer thickness in normal Japanese eyes measured with optical coherence tomography. Jpn J Ophthalmol 2010;54:36-42.

22. Bendschneider D, Tornow RP, Horn FK, et al. Retinal nerve fiber layer thickness in normals measured by spectral domain OCT. J Glaucoma 2010;19:475-82.

23. Knight OJ, Chang RT, Feuer WJ, Budenz DL. Comparison of retinal nerve fiber layer measurements using time domain and spectral domain optical coherent tomography. Ophthalmology 2009;116:1271-7.

24. Sung KR, Kim DY, Park SB, Kook MS. Comparison of retinal nerve fiber layer thickness measured by Cirrus HD and Stratus optical coherence tomography. Ophthalmology 2009;116:1264-70, 1270.el.

25. Mikelberg FS, Drance SM, Schulzer M, et al. The normal human optic nerve. Axon count and axon diameter distribution. Ophthalmology 1989;96:1325-8. 